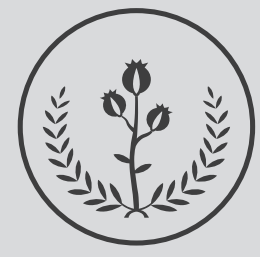

FUCS $x$

№2 . 2021

ISSN: 0121-7372 • ISSN electrónico: 2462-991X

\title{
Avances de las ciencias del movimiento desde un enfoque integrativo de las disciplinas de la salud y el bienestar
}

${ }^{a}$ Fisioterapeuta. Especialista en rehabilitación cardiopulmonar. Magister en Ciencias de la Actividad Física y Deporte. Doctor en Terapia Manual. Decano de la Facultad de Ciencias del Movimiento. Fundación Universitaria de Ciencias de la Salud. Bogotá DC, Colombia.

C 2021 Fundación Universitaria de Ciencias de la Salud - FUCS. Este es un artículo Open Access bajo la licencia CC BY-NC-ND (http://creativecommons.org/licenses/by-nc-nd/4.0/).

El movimiento humano es parte del conocimiento con un futuro promisorio frente a los desafíos actuales de las sociedades y del avance científico en los ámbitos de la salud y el bienestar de las personas, como son la promoción de la salud, la prevención de la enfermedad, la asistencia y la rehabilitación. La investigación conjugada con el emprendimiento permiten generar un dialogo de las disciplinas que componen las ciencias del movimiento, con una visión global e internacional de manera propositiva como lo define la proyección organizacional de la Fundación Universitaria de Ciencias de La Salud para el avance y crecimiento de la nueva Facultad de Ciencias del Movimiento. Se ha destacado el posicionamiento del programa de Fisioterapia desde 2018 cuando fue concebido con un enfoque hacia el área cardiorrespiratoria. Es allí donde se genera el funcionamiento $y$ funcionalidad requeridos para la planeación, ejecución y transformación del movimiento en los seres humanos a través del engranaje de los sistemas cardiovascular, pulmonar y mitocondrial de la fibra muscular, constituyendo una triada para la generación de energía. La expresión del movimiento facilita su comprensión teórica en cuanto al control y la organización, así como de los cambios como función del desarrollo, el aprendizaje y la rehabilitación. Las ciencias del movimiento permiten vislumbrar campos de conocimiento a partir de la investigación teórica, para emprender estudios aplicados en la educación del movimiento de las personas, la actividad física, el deporte, la danza y la rehabilitación. Así la Facultad de Ciencias del Movimiento emerge del campo de la fisioterapia como parte de los procesos teóricos adelantados en la profesión, reconociendo que las relaciones entre la salud y el movimiento han sido los dos elementos fundamentales en su desarrollo, siendo pertinente describir y ampliar las categorías que pueden fortalecer los enfoques y tendencias de la disciplina.

\section{INFORMACIÓN DEL ARTÍCULO}

Historia del artículo: Fecha recibido: mayo 27 de 2021 Fecha aceptado: mayo 28 de 2021
Autor para correspondencia. Jorge Enrique Moreno Collazos jemorenol@fucsalud.edu.co
DOI

10.31260/RepertMedCir.01217372.1229 


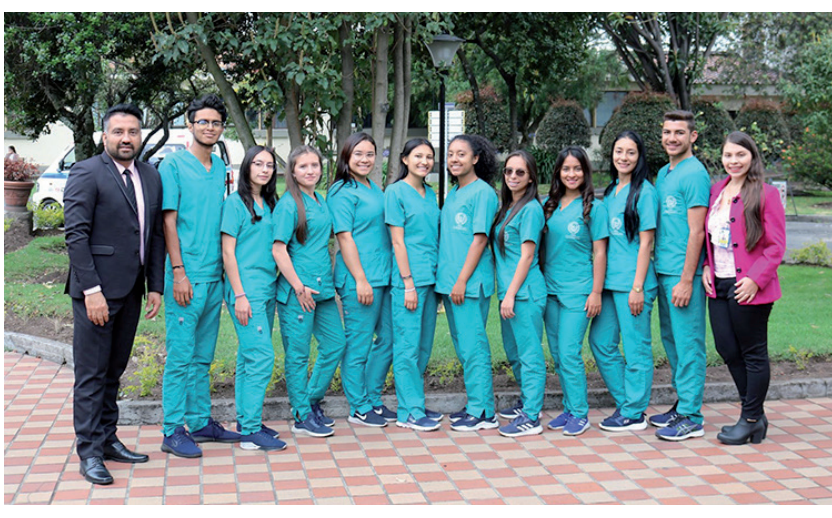

Figura 1. Semillero Movimiento y Salud. Fundación Universitaria de Ciencias de la Salud.

En la actualidad existe un énfasis en la comprensión de la fisioterapia desde el campo no sólo biológico, sino también social. Esta fusión, aunque incipiente, se orienta a incorporar nuevas categorías a la mirada biológica hegemónica, observando dos vertientes: la primera basada en una concepción funcionalista del movimiento y por tanto del papel de la fisioterapia en el cuidado de esa capacidad y en la atención a la desviación de los patrones de normalidad, y la segunda centrada en aproximar los elementos sociales y en definir que la actuación fisioterapéutica trasciende el control de la alteración funcional y biológica del movimiento corporal.

La relación entre la salud y la globalización, aunque remite a la salud internacional, le imprime nuevas transformaciones y una dinámica difícil de predecir. Los cambios que se están suscitando alrededor de estos temas han alcanzado tal ímpetu que están llevando al nuevo enfoque de "salud global". Esta área se basa en las competencias y funciones del concepto de la salud pública como parte de la política social e inclusión hacia el respeto y protección de los derechos de los ciudadanos, la equidad en salud y la gobernanza de la salud pública como principios rectores de las personas en situación de discapacidad.

Hoy, gran parte de la actividad en fisioterapia y robótica de rehabilitación se ha centrado en la capacidad de rehabilitar el movimiento de las personas que han sufrido un evento que afecta su calidad de vida. Las principales razones de este énfasis son: a) un número relativamente grande de pacientes en estas condiciones, b) los altos costos asociados con la rehabilitación y c) el grado de avance que los pacientes pueden experimentar con una rehabilitación intensiva como lo es actualmente la atención en las unidades de cuidado intensivo del síndrome poscuidado COVID-19, cuya asistencia y rehabilitación cardiopulmonar es liderada por los fisioterapeutas bajo el énfasis cardiorrespiratorio, articulado con un enfoque de la realidad virtual y los procesos de telerrehabilitación que nos permiten estar a la vanguardia de la salud y bienestar de las personas, mediante la simulación en laboratorios especializados para la formación de los futuros profesionales.

Lo anterior, debe inducir a nuestros estudiantes de la Facultad de Ciencias del Movimiento a generar actividades de construcción disciplinar a partir de su espíritu investigativo y de transformación social que nace en el semillero Movimiento y Salud, donde se avanza en proyectos de investigación liderados por docentes, de acuerdo con su interés por participar y su responsabilidad y compromiso. La investigación formativa será una actividad académica que desarrolle el alumno en escenarios reales de acuerdo con las estrategias diseñadas para el futuro de las ciencias del movimiento, con miras a la generación de estudios de maestría y doctorado bajo la rigurosidad del método científico y así dar respuesta a las necesidades del objeto de estudio de las disciplinas que constituyen las ciencias del movimiento.

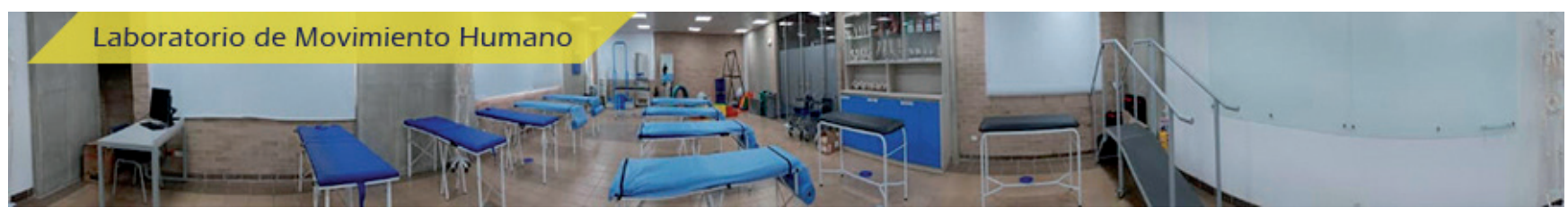

Figura 2. Laboratorio de Movimiento Humano 